\title{
Swelling-Based Chemical Sensing With Unmodified Optical Fibers
}

\author{
Alin JDERU ${ }^{1,2}$, Dorel DOROBANTU ${ }^{2}$, Dominik ZIEGLER ${ }^{1}$, and \\ Marius ENACHESCU ${ }^{2,3 *}$
}

\author{
${ }^{1}$ S.C. NanoPro START MC S.R.L., Bucharest 033091, Romania \\ ${ }^{2}$ Center for Surface Science and Nanotechnology, University Politehnica of Bucharest, Bucharest 060042, Romania \\ ${ }^{3}$ Academy of Romanian Scientists, Bucharest 050094, Romania \\ ${ }^{*}$ Corresponding author: Marius ENACHESCUＩ-mail: marius.enachescu@cssnt-upb.ro
}

\begin{abstract}
We use distributed fiber optic strain sensing to examine swelling of the fiber's polymer coating. The distributed sensing technique that uses unmodified low-cost telecom fibers opens a new dimension of applications that include leak detection, monitoring of water quality, and waste systems. On a short-range length scale, the technology enables "lab-on-a-fiber" applications for food processing, medicine, and biosensing for instance. The chemical sensing is realized with unmodified low-cost telecom optical fibers, namely, by using swelling in the coating material of the fiber to detect specific chemicals. Although generic and able to work in various areas such as environmental monitoring, food analysis, agriculture or security, the proposed chemical sensors can be targeted for water quality monitoring, or medical diagnostics where they present the most groundbreaking nature. Moreover, the technique is without restrictions applicable to longer range installations.
\end{abstract}

Keywords: Chemical sensing; distributed fiber optic strain; short range sensing; Brillouin optical time domain analysis

Citation: Alin JDERU, Dorel DOROBANTU, Dominik ZIEGLER, and Marius ENACHESCU, "Swelling-Based Chemical Sensing With Unmodified Optical Fibers," Photonic Sensors, 2022, 12(2): 99-104.

\section{Introduction}

Distributed fiber optic sensing has found applications in communications networks monitoring, structural health monitoring, shape sensing, pipeline and electrical transmission line monitoring, and intrusion detection for perimeter security applications [1, 2]. Fiber optic sensing exploits scattering of light in optical fibers to monitor mechanical perturbations, temperature changes, and strain. Unlike fiber Bragg gratings, which only provide information from discrete sensors locations, distributed fiber optic sensing collects information along the entire length of the fiber. We recently demonstrated that distributed temperature sensing can be used to monitor flow rates in a fluidic conduit [3]. Here, we demonstrate a distributed method for chemical sensing. The chemical sensing is based on swelling of the coating layer which axially strains the core of the glass fiber. Well established distributed strain sensing techniques, such as optical frequency-domain reflectometry (OFDR) or Brillouin optical time-domain analysis (BOTDA), can be utilized to detect the local changes in strain along the length of the fiber optic waveguide and thus indirectly

Received: 12 December 2020 / Revised: 25 May 2021

(C) The Author(s) 2021. This article is published with open access at Springerlink.com

DOI: $10.1007 / \mathrm{s} 13320-021-0637-2$

Article type: Regular 
measure the presence of chemicals. Since light is confined within the optical fiber core by total internal reflection, it is decoupled from the surroundings beyond the boundaries of the fiber cladding. Hence, the majority of fiber optical chemical sensing approaches $[4,5]$ only probe the chemical environment at the end of the optical fiber or at a few discrete locations where the light is purposefully guided into a sensing element. Since no light is lost in our configuration, thousands of such integrated sensors can be implemented along the same fiber optic line and read out simultaneously. This opens a new dimension for applications including leak detection and monitoring of water quality and waste systems. For instance, when utilized on a short length scale, the technology enables "lab-on-a-fiber" applications for food processing, medicine, or biosensing. On the large length scale (up to $100 \mathrm{~km}-200 \mathrm{~km}$ ), the approach enables highly scalable and cost effective quasi-distributed monitoring of environmental chemical state. Although generic, and able to work in various areas such as environmental monitoring, food analysis, agriculture, and security, the proposed chemical sensor can be tailored for water quality monitoring, leak detection, or medical diagnostics wherein they present the most groundbreaking contributions.

\section{Materials and methods}

\subsection{Principle of measurement}

We use standard telecom optical fibers, which consist of a core, a cladding, and a polymer coating with the dimensions as indicated in Fig. 1. Upon exposure to a chemical (I) the coating swells if the molecule can diffuse into the matrix of the polymer material (II). Swelling means a volume change of the coating which as illustrated can happen radially and axially. The axial swelling induces a swelling strain on the cladding and core of the fiber, which can be detected using OFDR or BOTDA based distributed strain sensing methods. Strain in unmodified optical fibers with a typical polyurethane coating were tested in different organic solvents. Their concentration in water can be detected within a few percent. The very first assumption that the chemical sensing is based on strain buildup by swelling of the coating, was validated by the fact that without any coating there was no strain build-up at all.

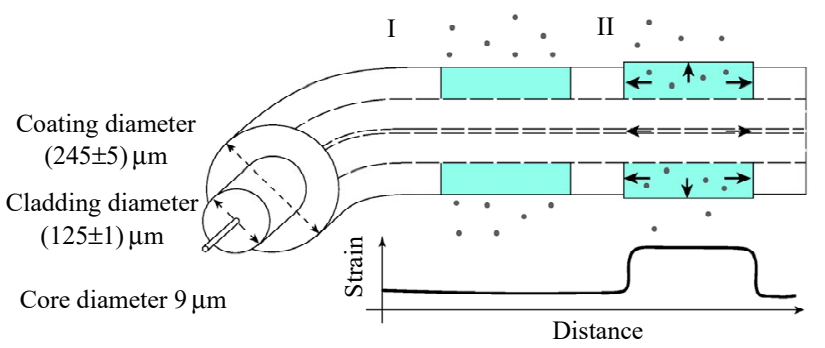

Fig. 1 Illustration of fiber optical chemical sensing with a standard single mode telecommunication fiber. The cross section shows the diameters of the glass core, glass cladding, and polymer coating. The dots illustrate an analyte (I) diffusing into the coating of the fiber (II) and thus as the coating swells applies tensile strain on the cladding and core. Distributed fiber optical strain detection methods (such as BOTDA or OFDR) can monitor the locally induced strain and thereby indirectly detect the presence of an analyte.

\subsection{Distributed strain measurement}

Distributed fiber optic strain measurements can be done in the time domain or frequency domain. In time domain reflectometry, a short laser pulse is sent into the fiber and the backscattered light is analyzed as the pulse propagates along the fiber. The delay, i.e., the time it takes for the backscattered light to return, determines the location of scattering events. BOTDA [6,7] relies on scattering of light by an acoustic wave due to a non-elastic interaction with the acoustic phonons [8]. The limiting factor Brillouin based detection is the extremely low intensity of the scattered light and the highest spatial resolution of about $1 \mathrm{~m}$. These limitations can be overcome by frequency domain techniques such as OFDR. In coherent OFDR the wavelength is swept, and the light is coupled into an interferometer. The resulting interference signal contains information on the precise location and magnitude of any reflective events along the length of the fiber under test (FUT). Since measurements are obtained by sweeping the laser wavelength, the scattering amplitude corresponds to the amplitude of the frequency 
response of the fiber. Using a fast Fourier transform (FFT), the response along the sensing fiber is obtained. The local Rayleigh spectrum, required to extract the strain, is then obtained by calculating the inverse FFT (IFFT) of a windowed Rayleigh response over the fiber location. Finally, the obtained local Rayleigh spectrum is compared to a reference spectrum measured at known temperature and strain conditions. For more details on the implementation refer to [9] and [10].

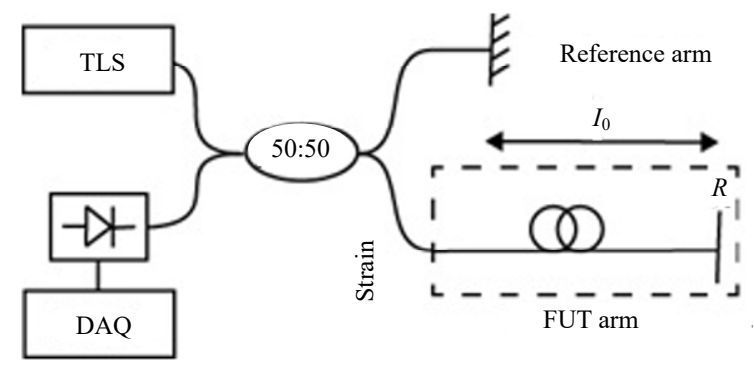

Fig. 2 Schematic setup of a standard OFDR system.

A standard layout of an OFDR setup is presented in coherent OFDR, which enables robust and practical distributed temperature measurements with sub-millimeter spatial resolution over tens to hundreds of meters of fiber with a temperature resolution below one Kelvin. This performance even at fast readout rates let us decide to use OFDR for our first demonstration on a $\sim 2$-m-long laboratory setup. For the strain measurement, we use a commercially available OFDR system (ODiSI-B by Luna Technologies Inc.). The spectral "fingerprint" of the Rayleigh backscatter may be thought of as a weak Bragg grating with a randomly varying period. Changes in effective line spacing and index of refraction shift the grating's resonant wavelength. The spectrum of the Rayleigh backscatter pattern responds in the same manner: changes in refractive index or physical length shift the reflected spectrum in frequency.

For the results shown in Fig. 3, a standard telecom optical fiber with an extremely low cost $[\sim \$ 0.10$ per meter, Corning SMF-28 (T)] is used. To test the swelling strain response in different solutions we make a simple setup to partially expose the fiber in the three baths [See Fig. 3(a)]. This allows us to simultaneously measure the chemical response to three different solvents in one experiment. In this experiment we chose ethanol, acetone, and isopropyl alcohol (IPA) since those three polar solvents are miscible in water, and are not very hazardous and typically used for cleaning purposes in many laboratories.

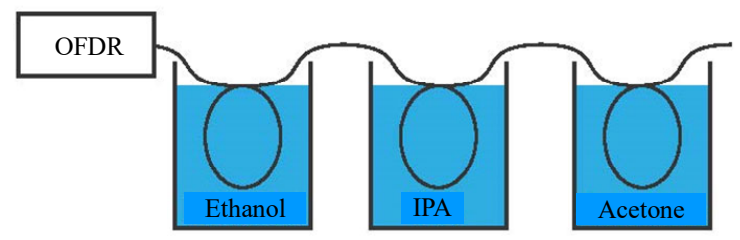

(a)

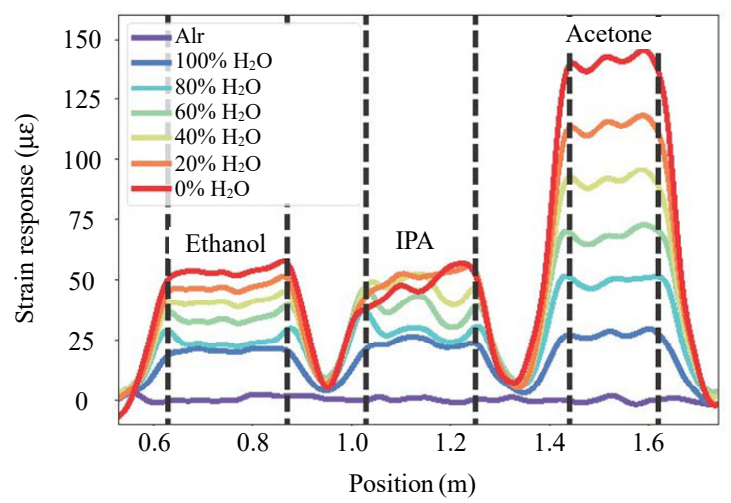

(b)

Fig. 3 Experimental setup and related results: (a) experimental setup where a single fiber is immersed in three solvents and the local swelling strain response is monitored using an OFDR interrogator and (b) swelling strain responses for different concentrations of ethanol, isopropyl alcohol (IPA), and acetone in water are measured on a two-meter-long fiber. The about 25-cm-long exposed areas show an increased swelling strain build-up with increased concentrations of the organic solvents measured after $15 \mathrm{~min}$ of exposure.

\section{Results}

We start with all baths empty and the setup equilibrated at ambient temperature, and record a baseline for all strain recordings [See air curve (purple) in Fig.3(b)]. The strain recordings are done at $4 \mathrm{~Hz}$. With a lateral resolution of $0.67 \mathrm{~mm}$, each recording contains 1800 data points along an about 2-m-long fiber. We then fill all containers with water, 
for which we can see a swelling strain of about $20 \mu \varepsilon$. The water reaches thermal equilibrium at room temperature before they are poured into the liquid containers where about $20-\mathrm{cm}$-long sections of the fiber were immersed. By gently touching the fiber with the fingertips that act as a local heat source, we are able to localize the exact positions on the fibers [see dotted lines in Fig. 3(b)]. The strain recording in air is used as a baseline measurement and after filling all three containers with water we observe an increase in the strain reaching up to $20 \mu \varepsilon$ (micro epsilon). (See dark blue curve). $1 \mu \varepsilon$ corresponds to an elongation of $0.0001 \%$, or a change in length of one micrometer per 1-m-long fiber. The time constants of the swelling are on the order of $2 \mathrm{~min}-$ $3 \mathrm{~min}$. In the following, we gradually increase the organic solvent contents in each of the three baths, in increments of 20 volume percent every $15 \mathrm{~min}$. The waiting time ensures that a steady state is reached before taking each measurement. The results for ethanol, IPA, and acetone for a volume percent in water of $0 \%, 20 \%, 40 \%, 60 \%, 80 \%$, and $100 \%$ are shown in Fig. 2(b), which plots the measured strain against the position along the fiber. We observe the highest strains of up to $150 \mu \varepsilon$ with acetone while ethanol and IPA reach only about $55 \mu \varepsilon$. Interestingly, the strain did not increase when going from $80 \%$ to $100 \%$ concentration in IPA, which might be explained by partial dissolution of the fiber material or delamination of the coating from the fiber that is leading to a relaxation and thus less observed strain. For the ethanol concentration from $20 \%$ to $100 \%$, we observe more pronounced peaks in the strain, which are decaying with higher concentration. The origin might be related to evaporation at the solvent air interface, and the related liquid meniscus. The origin of the slightly different strains resulting in a wave like pattern along the fiber is unclear. The thickness of the coating resulting in a diameter of $245 \mu \mathrm{m}$ can vary by $\pm 5 \mu \mathrm{m}$ that could be one potential explanation [11].
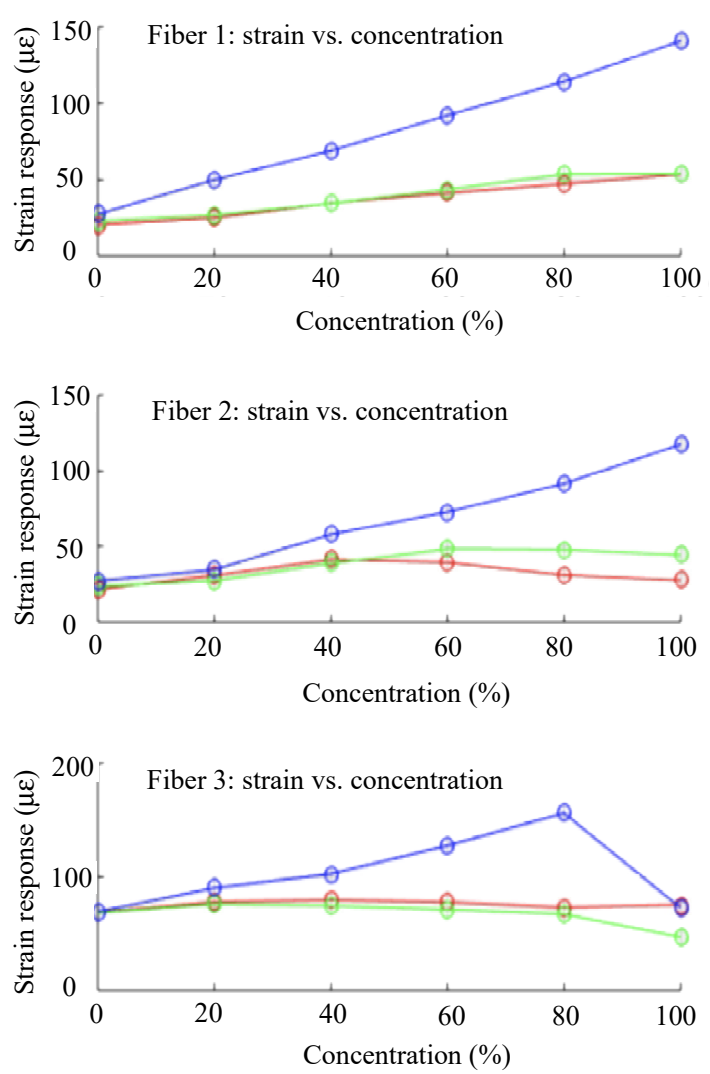

Fig. 4 Average swelling strain for three different unmodified telecommunication fibers with increasing volumetric concentrations of ethanol (red), isopropyl alcohol (green), and acetone (blue) in water.

We then test three different coatings on SMA-28 fibers and observe their strain buildup when immersing in different concentrations of the three solvents, as shown in Fig. 4. The three tested fibers are all manufactured by Corning: Fiber 1 is a Corning SMF-28 (T), Fiber 2 is a Corning SMF-28 [R fiber CPC6)], and Fiber 3 is a Corning SMF-28 (1300-1550-9-125-0.125F L-White). When the core and cladding are identical, the different responses indicate that the composition of the fiber's coating is different. For Fiber 1, we observe a monotonous nearly linear increase in the strain for all solvents, and only at $100 \%$ IPA we observe a slightly lower strain than that at $80 \%$. As described above, we attribute this slightly lower strain at $100 \%$ to partial dissolution or delamination of the coating, and for Fiber 2 this effect is much more pronounced, while for acetone strain increases with concentration. 


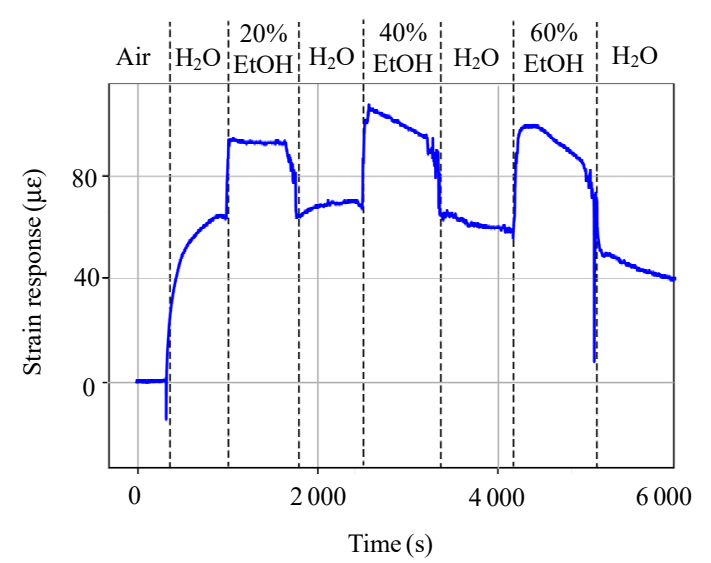

(a)

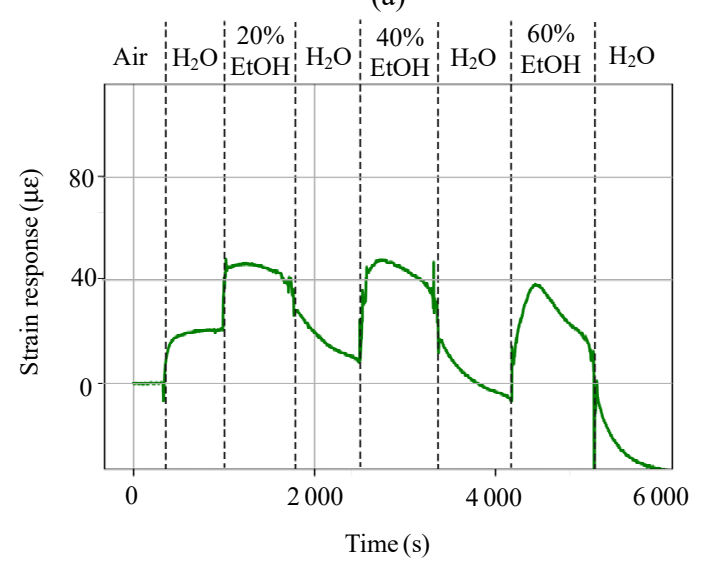

(b)

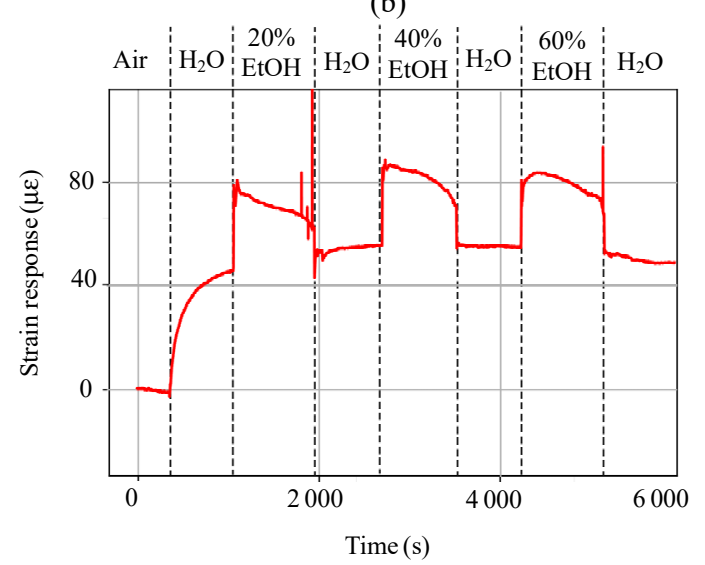

(c)

Fig. 5 Sequential exposure to water and ethanol/water mixtures the Rayleigh frequency shift and corresponding strain is shown for (a) Fiber 1, (b) Fiber 2, and (c) Fiber 3. Clearly, Fiber 2 does not withstand the long-term exposure. Upon returning to water after exposure to $20 \%$ ethanol for $10 \mathrm{~min}$ the strain reduces and eventually even becomes negative.

The strain plateaus at about $50 \mu \varepsilon$ above $50 \%$ concentration for IPA and even decreases for ethanol. For Fiber 3, the water induced strain (at $0 \%$ concentration of alcohols) is $80 \mu \varepsilon$ which is significantly larger than what the $20 \mu \varepsilon$ observed for Fibers 1 and 2. With increased concentration the strain only increases for acetone, but even drops for IPA and ethanol. These results illustrate that the sensing by polymer swelling is limited by possible delamination of the polymer film. Swelling and shrinking introduce a shear force at the sensor/polymer interface that eventually severs the covalent bonds holding the polymer layer onto the substrate. From the slopes of the measured swelling-induced strain of Fiber 1, one can infer a sensitivity of about $0.33 \mu \varepsilon / \%, \quad 0.40 \mu \varepsilon / \%$, and $1.15 \mu \varepsilon / \%$ for ethanol, IPA, and acetone, respectively. The limits of detection are given by the noise level of the used OFDR detection method which is about $0.1 \mu \varepsilon$ at the used bandwidth of $4 \mathrm{~Hz}$. Lateral or temporal averaging can lower this detection significantly.

In the next experiment we start with air to measure a baseline and then test the three fibers in 10 -min-long exposures to water followed by $20 \%$ ethanol, water, $40 \%$ ethanol, water, $60 \%$ ethanol, and water again. Consistent with the experiments shown in Fig. 4, we observe that Fiber 3 shows the most stable performance with no significant relaxation of strain over the time frame of $90 \mathrm{~min}$ (see Fig.5). In contrast, Fiber 2 shows a substantial drop in strain when switching from ethanol back to water. This can be evidence of delamination or partial dissolution of material even resulting in a transition from compressive to a more tensile strain compared to the initial conditions.

\section{Conclusions}

Our chemical sensing technique is based on sensing swelling strain, which leave the core of the optical fiber intact and therefore enable simultaneous readout of many sensing elements over long distances. We confirm that the observed strain in fact result from swelling of the coating by testing uncoated fibers, i.e., fibers where the coating is removed, which shows no strain response when immersing in any of the alcohols. Readout of multiple integrated sensors increases the reliability and reduces the measurement uncertainty over single sensor experiments. Although generic and able to work in various areas such as environmental monitoring, food analysis, agriculture, and security, 
the proposed chemical sensors can be targeted for water quality monitoring, or medical diagnostics where they present the most groundbreaking nature. Our results show that fibers with different coatings respond differently to exposure to different solvents. For exposure to ethanol, IPA, and acetone, we observe strains ranging from $20 \mu \varepsilon$ to $150 \mu \varepsilon$. For a large range of concentrations, the strain increases monotonously with the concentration of the solvent in water. While Fiber 3 shows good stability over time, the results with Fibers 1 and 2 show a decrease in strain over the testing period of $100 \mathrm{~min}$. Clearly, more research is needed to identify better suited coatings which adhere well to the optical fiber and ideally respond selectively to different analytes. Simultaneous readout of multiple integrated sensors would increase the reliability and reduce the measurement uncertainty over single sensor experiments. In addition to the strain value, one could also infer the solvation/reaction kinetics from the time constant of the strain change. This information might help to better identify specific reaction pairs.

We demonstrate this novel technique for short-range sensing, however, without difficulties this chemical sensing method is applicable with other fiber sensing techniques, e.g., BOTDA, the range can exceed $100 \mathrm{~km}$. Therefore, this technique can find use in leak detection in pipelines or any type of supply lines for chemical, medical, food or pharmaceutical industries. In the other extreme, using micro-fabricated optical waveguides instead of optical fibers could lead to miniaturization of such sensors.

\section{Acknowledgment}

We would like to thank Sina Sedighi and Prof. Marcelo Soto for fruitful discussions and for help with signal processing. This work was financed by the Romanian Ministry of Education and Research (Grant No. 34/01.09.2016, ID: P_37_788, MySMIS: 103364), project co-funded by the European Regional Development Fund through the Competitiveness Operational Program.
Open Access This article is distributed under the terms of the Creative Commons Attribution 4.0 International License (http://creativecommons.org/licenses/by/4.0/), which permits unrestricted use, distribution, and reproduction in any medium, provided you give appropriate credit to the original author(s) and the source, provide a link to the Creative Commons license, and indicate if changes were made.

\section{References}

[1] B. Lee, "Review of the present status of optical fiber sensors," Optical Fiber Technology, 2003, 9(2): $57-79$.

[2] X. Bao and L. Chen, "Recent progress in distributed fiber optic sensors," Sensors, 2012, 12(7): 86018639.

[3] A. Jderu, M. Enachescu, and D. Ziegler, "Mass flow monitoring by distributed fiber optical temperature sensing," Sensors, 2019, 19(19): 4151.

[4] P. Rivero, J. Goicoechea, and F. Arregui, "Optical fiber sensors based on polymeric sensitive coatings," Polymers, 2018, 10(3): 280.

[5] M. Yin, B. Gu, Q. F. An, C. Yang, Y. L. Guan, and K. T. Yong, "Recent development of fiber-optic chemical sensors and biosensors mechanisms, materials, micro/nano-fabrications and applications," Coordination Chemistry Reviews, 2018, 376: 348-392.

[6] T. Horiguchi and M. Tateda, "Optical- fiberattenuation investigation using stimulated Brillouin scattering between a pulse and a continuous wave," Optics Letters, 1989, 14(8): 408-410.

[7] T. Kurashima, T. Horiguchi, and M. Tateda, "Distributed-temperature sensing using stimulated Brillouin scattering in optical silica fibers," Optics Letters, 1990, 15(18): 1038-1040.

[8] X. Bao, J. Dhliwayo, N. Heron, D. J. Webb, and D. A. Jackson, "Experimental and theoretical studies on a distributed temperature sensor based on Brillouin scattering," Journal of Lightwave Technology, 1995, 13(7): 1340-1348.

[9] S. T. Kreger, D. K. Gifford, M. E. Froggatt, B. J. Soller, and M. S. Wolfe, "High resolution distributed strain or temperature measurements in single- and multi-mode fiber using swept-wavelength interferometry," Optical Fiber Sensors 2006, Cancun, Mexico, October 23-27, 2006.

[10] D. K. Gifford, S. T. Kreger, A. K. Sang, M. E. Froggatt, R. G. Duncan, M. S. Wolfe, et al., "Sweptwavelength interferometric interrogation of fiber Rayleigh scatter for distributed sensing application," Fiber Optic Sensors and Applications V. SPIE, 2007, 6770: 6770F.

[11] Corning SMF-28 CPC6 Single-mode optical fiber datasheet. http://www.iet.unipi.it/m.luise/Corning SMF28.pdf. 